\title{
A Study of Discrete-Time Optimum Current Controller for the Virtual Synchronous Generator under Constraints
}

\author{
Atushi Umemura, Rion Takahashi, Junji Tamura \\ Department of Electrical and Eslectronic Engineering, Kitami Institute of Technology, Kitami, Japan \\ Email: umemura@mail.kitami-it.ac.jp
}

How to cite this paper: Umemura, A., Takahashi, R. and Tamura, J. (2020) A Study of Discrete-Time Optimum Current Controller for the Virtual Synchronous Generator under Constraints. Smart Grid and Renewable Energy, 11, 1-20. https://doi.org/10.4236/sgre.2020.111001

Received: December 28, 2019

Accepted: January 24, 2020

Published: January 27, 2020

Copyright $\odot 2020$ by author(s) and Scientific Research Publishing Inc. This work is licensed under the Creative Commons Attribution International License (CC BY 4.0).

http://creativecommons.org/licenses/by/4.0/

\begin{abstract}
In recent years, power generation using renewable energy sources has been increasing as a solution to the global warning problem. Wind power generation can generate electricity day and night, and it is relatively more efficient among the renewable energy sources. The penetration level of variable-speed wind turbines continues to increase. The interconnected wind turbines, however, have no inertia and no synchronous power. Such devices can have a serious impact on the transient stability of the power grid system. One solution to stabilize such grid with renewable energy sources is to provide emulated inertia and synchronizing power. We have proposed an optimal design method of current control for virtual synchronous generators. This paper proposes an optimal control method that can follow the virtual generator model under constrains. As a result, it is shown that the proposed system can suppress the peak of the output of semiconductor device under instantaneous output voltage drop.
\end{abstract}

\section{Keywords}

Wind Energy Generation, Virtual Synchronous Generator, Electric Current

Control, Optimal Control, Discreate-Time Model Following Control,

Multi-Objective Genetic Algorithm

\section{Introduction}

At present day, the introduction of renewable energy that can suppress global warming and gas emissions that causes global warming is progressing. Renewable energy includes "solar power", "wind power", "ocean energy", "hydropower", "geothermal", and "biomass", etc. In particular, wind power can generate electricity day and night and it is a relatively efficient power generation method 
among the renewable sources. According to the Global Wind Report 2018, the installed amount of wind power in the world was $540 \mathrm{GW}$ in 2017 and $591 \mathrm{GW}$ in 2018, and the installed amount is increasing [1]. In Japan, the amount of renewable energy introduced was 51 GW in FY2019 [2] (FY is fiscal year in Japan). In solar power generation and wind power generation, grid-connected inverters are used to interconnect the fluctuating output power to the power system.

In [3], a hybrid adaptive control approach is proposed for the VS-VP half-direct driven wind energy conversion systems in which a combined control is adopted between pitch angle control and variable generator torque regulation in different operating regions. Both the generator torque and pitch angle are controlled in partial or full load regions. Furthermore, an effective nonlinear PID pitch controller is proposed to track the pitch angle reference command in the full load region of operation.

In [4], the artificial neural network based direct torque control strategy and direct power control strategy are proposed for permanent magnet synchronous generator-based wind power system.

There are many studies applying such advanced control methods to efficiently obtain wind power.

Generally, synchronous generators are used in power systems to supply electrical energy. The inertia and synchronization power of the synchronous generator contribute to the stability of the commercial power system. On the other hand, it is pointed out that grid-connected inverters usually have no inertia and no synchronizing power, and thus, if the penetration level of renewable energy increases, the ability to stabilize the power system may be insufficient.

As a solution to this problem, a method has been proposed in which inertia and synchronous power are virtually provided to the grid-connected inverter [5] [6] [7]. The virtual synchronous generator is a method that provides functions of synchronous power and virtual inertia to interconnected inverters [5]. This method is called an asynchronous virtual machine or a virtual synchronous generator. The Virtual Synchronous Generator research group focused on a current-reference emulated inertia provided by a phase lock loop (PLL) for the control of inertia on a rotating dq frame in 2007. The Institute of Electrical Power Eng. (IEPE) virtual synchronous generator (Clausthal University of Technology in Germany) is based on simplified synchronous generator model. This method helps to improve the frequency stabilization in short time.

Application of the virtual synchronous generator control to a wind diesel system with energy storage system (ESS) has been proposed in [6]. Their ESS contributes to the frequency stability of small scale grid systems under renewable energy and load change. The virtual inertia method with optimized variable inertia can improve the grid frequency response with lower power compared to the fixed virtual inertia method.

In [7], a simple model of grid-forming and grid-following virtual inertia emulation to mimic the behavior of synchronous machines are developed for fast 
frequency response in weak grid system.

As a result, the interconnected inverter of each wind power generator is expected to have the inertia and the synchronous power virtually and supplement the lack of inertia and the synchronizing power of the system. A converter with virtual inertia control uses current control (voltage control) to realize the virtual inertia. In the previous researches, PI control is often used after converting to a rotating coordinate system. An optimal current control method was proposed in [8]. The new method proposed in this paper has better tracking ability than the conventional method. Therefore the reactive power fluctuation can be suppressed.

Such equipment typically requires constraint to prevent excess output over the rated capacity in the event of a grid system failure. If such a system demands strict control performance without considering the saturation of the control input, the control performance may be degraded or unstable. To deal with such problems, a model-recovery-anti-windup compensator scheme of the electro hydro servo-system is proposed to suppress the control saturation caused by external load disturbance and modeling uncertainly [9]. On the other hand, there is research on anti-windup control system design using LMI [10]. This shows that solvable conditions can be derived in the form of LMI conditions for the stabilization problem of distributed anti-windup control systems with input saturation. This method is to provide optimal control with restrictions analytically, but there is a problem in realizing it in real time because large amount of calculation is required to derive the control law.

In previous research, we have proposed discrete-time model following control for optimal current control that changes the state feedback gain for each sample time for a virtual synchronous generator model with a inertia and synchronous power [8]. Since this control system uses instantaneous value control, the tracking error is small, and it can be expected that the reactive power fluctuation caused by the current control error is smaller than that of the conventional PI control method.

In this paper, we propose an optimal current control method with suppressed inverter side filter current, the inverter side filter voltage, and the inverter side filter power, and consider the optimized weight of the evaluation function.

This paper describes the proposed control system for the grid-connected wind power generator system in Chapter 2. Chapter 3 describes the simulation result. Finally, we summarize the results in Chapter 4.

\section{Proposed Current Control System}

\subsection{Interconnected Wind Turbine System}

We consider a large-scale wind power generator system with a grid-connected inverter as shown in Figure 1.

A wind generator is connected to the grid via AC/DC converter, grid-connected inverter, LCL filter, and grid-connected transformer. 


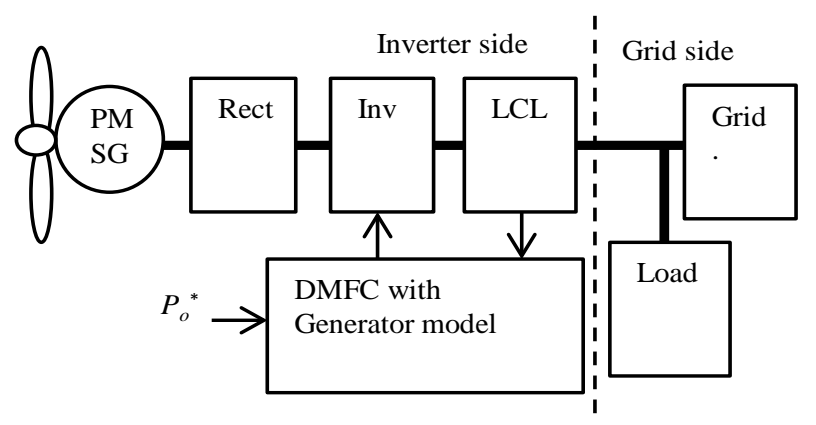

Figure 1. Wind turbine system with grid connected inverter.

In this paper, we focus on the interconnected inverter and the system, assuming that the wind turbine of the wind generator is rotating under the rated constant wind speed.

\subsection{Interconnected Inverter with LCL Filter}

An equivalent circuit of the interconnected inverter with an LCL filter connected to the system is shown (see Figure 2).

Since this inverter is connected to a balanced three-phase power system, Figure 2 shows a circuit for one phase.

The grid system model is composed of equivalent system impedance and infinite bus. The DC link capacitor and the full-bridge inverter are simulated with an ideal voltage source and are connected to the system via an LCL filter. The following differential equation is defined for the system model to which the interconnection inverter is connected, as the control plant model.

$$
\begin{gathered}
\frac{\mathrm{d}}{\mathrm{d} t} i_{p o}+T_{g d}^{-1} i_{p o}=l_{g d}^{-1}\left(v_{o}-v_{g}\right) . \\
\frac{\mathrm{d}}{\mathrm{d} t} i_{p o}+T_{2}^{-1} i_{p o}=l_{2}^{-1}\left(v_{c}-v_{o}\right) . \\
\frac{\mathrm{d}}{\mathrm{d} t} i_{i}+T_{1}^{-1} i_{i}=l_{1}^{-1}\left(v_{i}-v_{c}\right) . \\
\frac{\mathrm{d}}{\mathrm{d} t} v_{c}=c_{Y}^{-1}\left(i_{i}-i_{p o}\right) .
\end{gathered}
$$

where, the filter output current vector $i_{p o}$ is composed of zero-phase, negative-phase, and positive-phase components of the three-phase current, and each component is an instantaneous current normalized by the rated current. Similarly, $i_{i}$ is the input current vector of the LCL filter. $v_{c}$ standardizes the instantaneous value vector of the star-connected capacitance voltage normalized by the rated voltage. Similarly, $v_{i}$ is the filter input voltage vector, $V_{o}$ is the output voltage vector, and $\mathrm{V}_{g}$ is the infinite bus voltage vector. $C_{Y}$ is the matrix of star-connected capacitances multiplied by the rated impedance, and $l_{1}$ and $l_{2}$ are the input and output inductance matrices of the filter normalized by the rated impedance. $T_{1}$ and $T_{2}$ are the input and output time constant matrices of the filter. The above matrix is defined by the following equation. 


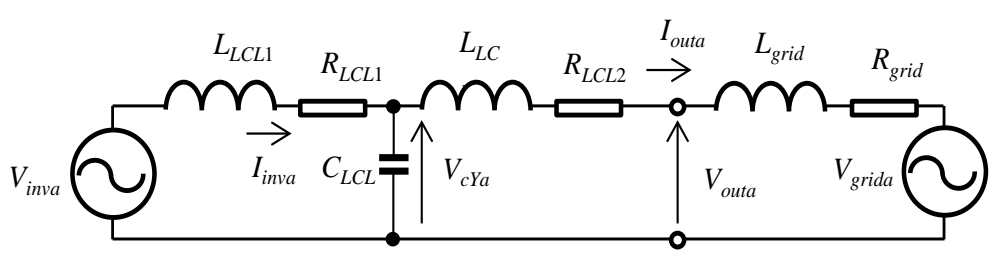

Figure 2. Grid system and connected inverter.

$$
\begin{gathered}
i_{p o}=\frac{3 V_{\text {baseY }}}{S_{\text {base }}} C_{\alpha \beta}^{a b c}\left[\begin{array}{c}
I_{\text {outa }} \\
I_{\text {outb }} \\
I_{\text {outc }}
\end{array}\right], i_{i}=\frac{3 V_{\text {baseY }}}{S_{\text {base }}} C_{\alpha \beta}^{a b c}\left[\begin{array}{c}
I_{\text {inva }} \\
I_{\text {invb }} \\
I_{\text {invc }}
\end{array}\right] \\
V_{c}=\frac{1}{V_{\text {baseY }}} C_{\alpha \beta}^{a b c}\left[\begin{array}{c}
V_{c Y a} \\
V_{c Y b} \\
V_{c Y c}
\end{array}\right], v_{o}=\frac{1}{V_{\text {baseY }}} C_{\alpha \beta}^{a b c}\left[\begin{array}{c}
V_{\text {outa }} \\
V_{\text {outb }} \\
V_{\text {outc }}
\end{array}\right] \\
V_{g}=\frac{1}{V_{\text {baseY }}} C_{\alpha \beta}^{a b c}\left[\begin{array}{c}
V_{\text {grida }} \\
V_{\text {gridb }} \\
V_{\text {gridc }}
\end{array}\right], v_{i}=\frac{1}{V_{\text {baseY }}} C_{\alpha \beta}^{a b c}\left[\begin{array}{c}
V_{\text {inva }} \\
V_{\text {invb }} \\
V_{\text {invc }}
\end{array}\right] \\
l_{1}=\frac{S_{\text {base }}}{3 V_{\text {baseY }}^{2}} L_{L C L 1} C_{\alpha \beta}^{a b c-1} C_{\alpha \beta}^{a b c}, l_{2}=\frac{S_{\text {base }}}{3 V_{\text {bseY }}^{2}} L_{L C L 2} C_{\alpha \beta}^{a b c-1} C_{\alpha \beta}^{a b c} \\
l_{g d}=\frac{S_{\text {base }}}{3 V_{\text {bseY }}^{2}} L_{\text {grid }} C_{\alpha \beta}^{a b c-1} C_{\alpha \beta}^{a b c}, \quad C_{Y}=\frac{3 V_{\text {baseY }}^{2}}{S_{\text {base }}} C_{Y} C_{\alpha \beta}^{a b c-1} C_{\alpha \beta}^{a b c} \\
T_{1}^{-1}=L_{L C L 1}^{-1} R_{L C L 1} C_{\alpha \beta}^{a b c-1} C_{\alpha \beta}^{a b c}, T_{2}^{-1}=L_{L C L 2}^{-1} R_{L C L 1} C_{\alpha \beta}^{a b c-1} C_{\alpha \beta}^{a b c} \\
T_{g d}^{-1}=L_{\text {grid }}^{-1} R_{\text {grid }} C_{\alpha \beta}^{a b c-1} C_{\alpha \beta}^{a b c} .
\end{gathered}
$$

where, the rated capacity and rated voltage is the self-capacity of the virtual generator, the rated capacity is $S_{\text {base }}[\mathrm{VA}]$ and the rated phase voltage is $V_{\text {baseY }}[\mathrm{V}]$. The three-phase to two-phase conversion matrix is $C_{\alpha \beta}^{a b c}$, and the loss of the LCL filter is a scalar $R_{L C L 1}[\Omega]$ and $R_{L C L 2}[\Omega]$, Inverter-side inductance of LCL filter in scalaris $L_{L C L 1}[\mathrm{H}]$, Grid-side LCL filter inductance in scalaris $L_{L C L 2}[\mathrm{H}]$, Grid system inductance component in scalaris $L_{\text {grid }}[H]$, and the star-connected capacitance in scalar is $C_{L C L}[\mathrm{~F}]$. A balanced three-phase circuit is assumed, so the parameters of the three-phase circuit are the same for each phase.

The sum of Equations (1) and (2) gives the following equation:

$$
\left(l_{g d}+l_{2}\right) \frac{\mathrm{d}}{\mathrm{d} t} i_{p o}+\left(l_{g d} T_{g d}^{-1}+l_{2} T_{2}^{-1}\right) i_{p o}=\left(v_{c}-v_{g}\right) .
$$

The voltage vector of the infinite bus is given by the following equation.

$$
\begin{gathered}
\frac{\mathrm{d}}{\mathrm{d} t} v=\omega_{\text {base }} S_{\alpha \beta} v . \\
v_{g}=\left|v_{g}\right| v .
\end{gathered}
$$

where, $v$ is a unit vector of the system voltage. The magnitude of the system voltage $\left|v_{g}\right|\left(=C_{p v g}\right)$ is a constant scalar quantity. $\omega_{\text {base }}[\mathrm{rad} / \mathrm{sec}]$ is a scalar quantity 
and the rated system frequency, and $S_{\alpha \beta}$ is the following oblique matrix.

$$
S_{\alpha \beta}=\left[\begin{array}{ccc}
1 & 0 & 0 \\
0 & 0 & -1 \\
0 & 1 & 0
\end{array}\right] \text {. }
$$

Assuming that the input voltage vector of filter $v_{i}$ and the grid system voltage vector $V_{g}$ are constant at the time interval $T_{s}$, the values at the discrete time $k$ are defined as $V_{i k}$ and $v_{g k}$. Then, Equations (3) to (8) can be represented by the following discrete-time equations.

$$
\begin{gathered}
i_{p o k+1}=A_{p i o} i_{p o k}+B_{p i o}\left(v_{c k}-v_{g k}\right) . \\
i_{i k+1}=A_{p i i} i_{i k}+B_{p i i}\left(v_{i k}-v_{c k}\right) . \\
v_{c k+1}=v_{c k}+B_{p v c}\left(i_{i k}-i_{p o k}\right) . \\
v_{k+1}=A_{p v g} v_{k} . \\
v_{g k}=C_{p v g} v_{k} .
\end{gathered}
$$

where $i_{p o k}, V_{c k}, i_{i k}$, and $v_{k}$ are discrete-time vectors at $k$-th time of the continuous time vector $i_{p o}, v_{\mathcal{s}} i_{i}$ and $v$ at the time interval $T_{s}, A_{p i o}, A_{p i i}$ and $A_{p v g}$ are the transition matrices obtained from the differential Equations (6), (3), and (4), respectively, and are given as follows.

$$
\begin{gathered}
A_{\text {pio }}=\exp \left\{-T_{s}\left(l_{g d}+l_{2}\right)^{-1}\left(l_{g d} T_{g d}^{-1}-l_{2} T_{2}^{-1}\right)\right\} \\
A_{p i i}=\exp \left\{-T_{s} T_{1}^{-1}\right\} A_{p v g}=\exp \left\{-T_{s} \omega_{\text {base }} S_{\alpha \beta}\right\} \\
B_{\text {pio }}=\left\{\int_{0}^{T_{s}} \exp \left\{-\left(T_{s}-\xi\right)\left(l_{g d}+l_{2}\right)^{-1}\left(l_{g d} T_{g d}^{-1}-l_{2} T_{2}^{-1}\right)\right\} \mathrm{d} \xi\right\}\left(l_{g d}+l_{2}\right)^{-1} \\
B_{p i i}=\left\{\int_{0}^{T_{s}} \exp \left\{-\left(T_{s}-\xi\right) T_{1}^{-1}\right\} \mathrm{d} \xi\right\} l_{1}^{-1} \\
B_{p v c}=\left\{\int_{0}^{T_{s}} \exp \left\{T_{s}-\xi\right\} \mathrm{d} \xi\right\} c_{Y}^{-1}
\end{gathered}
$$

By combining Equations (10) to (14), the equation of the controlled model can be expressed as follows.

$$
\left[\begin{array}{c}
x_{p 1 k+1} \\
v_{k+1}
\end{array}\right]=\left[\begin{array}{cc}
A_{p d 11} & A_{p d 12} \\
0 & A_{p v g}
\end{array}\right]\left[\begin{array}{c}
x_{p 1 k} \\
v_{k}
\end{array}\right]+\left[\begin{array}{c}
B_{p d 1} \\
0
\end{array}\right] V_{i k} .
$$

where, the state vector is composed of $x_{p 1 k}=\left[i_{p o k}^{\mathrm{T}}, v_{c k}^{\mathrm{T}}, i_{i k}^{\mathrm{T}}\right]^{\mathrm{T}}$ and $v_{k}$ and the input vector is the filter input voltage vector $v_{i k}$.

$$
A_{p d 11}=\left[\begin{array}{ccc}
A_{p i o} & B_{p i o} & 0 \\
-B_{p v c} & 1 & B_{p v c} \\
0 & -B_{p i i} & A_{p i i}
\end{array}\right], A_{p d 12}=\left[\begin{array}{c}
-B_{p i o} C_{p v g} \\
0 \\
0
\end{array}\right], B_{p d 1}=\left[\begin{array}{c}
0 \\
0 \\
B_{p i i}
\end{array}\right] .
$$

The output voltage $v_{o}$ is given by the following equation from Equations (1) and (2).

$$
\left(l_{2}^{-1}+l_{g d}^{-1}\right) v_{o}=-\left(T_{2}^{-1}-T_{g d}^{-1}\right) i_{p o}+l_{2}^{-1} v_{c}+l_{g d}^{-1} v_{g}
$$


Therefore, the output voltage $v_{o k}$ at the time $k$ is obtained by the following equation.

$$
V_{o k}=\left[\begin{array}{ll}
C_{o 1} & C_{o 2}
\end{array}\right]\left[\begin{array}{ll}
x_{p 1 k}^{\mathrm{T}} & V_{g k}^{\mathrm{T}}
\end{array}\right]^{\mathrm{T}} .
$$

Therefore, from Equations (19) and (14), the output voltage is obtained from the state vectors $X_{p 1 k}$ and $v_{k}$.

\subsection{Virtual Synchronous Generator Model}

The swing equation of the virtual generator is given as follows.

$$
\frac{\mathrm{d}}{\mathrm{d} t} \omega_{g}+T_{g}^{-1} \omega_{g}=\omega_{\text {base }} M_{g}^{-1}\left(\tau_{i}-\tau_{o}\right) .
$$

Here, $M_{g}[\mathrm{~s}]$ is a scalar and is the inertia constant of the virtual generator (twice the stored energy constant). $T_{g}[\mathrm{~s}]$ is a scalar quantity and the time constant of the mechanical system, $\omega_{g}[\mathrm{rad} / \mathrm{sec}]$ is a scalar quantity and the rotation speed of the virtual synchronous generator, $\tau_{i}[\mathrm{pu}]$ is the input torque as a scalar quantity, $\tau_{o}[\mathrm{pu}]$ is the output torque as a scalar quantity. These to rquesare, when $P_{i}[\mathrm{pu}]$ is input as a scalar quantity and $P_{o}[\mathrm{pu}]$ is output as a scalar quantity, $\tau_{i}=P_{i} \omega_{\text {base }} / \omega_{g}, \tau_{o}=P_{o} \omega_{\text {base }} / \omega_{g}$. The rated rotor speed of the virtual synchronous generator $\omega_{\text {base }}[\mathrm{rad} / \mathrm{sec}]$ is equal to the grid rated frequency because the virtual synchronous generator is a 2-pole virtual synchronous generator.

The equivalent circuit equation of the virtual generator is as follows.

$$
\frac{\mathrm{d}}{\mathrm{d} t} i_{v o}+T_{v o}^{-1} i_{v o}=l_{g}^{-1}\left(e_{g}-v_{o}\right)
$$

where, $T_{v o}[\mathrm{~s}]$ is the time constant matrix of the electric system of the virtual generator, $l_{g}$ is the inductance matrix, $i_{v o}$ is the output current vector of the virtual generator, and $e_{g}$ is the no-load voltage vector. These vectors are composed of zero-phase, positive-phase, and negative-phase components in the grid-connected inverter system. $e_{g}$ is given by the following equation.

$$
\begin{aligned}
\frac{\mathrm{d}}{\mathrm{d} t} e & =\omega_{g} S_{\alpha \beta} e . \\
e_{g} & =\left|e_{g}\right| e .
\end{aligned}
$$

where, $e$ is the unit vector of the no-load voltage vector, and $\left|e_{g}\right|[\mathrm{pu}]$ is the magnitude of the no-load voltage. $\left|e_{g}\right|$ is given by the following equation.

$$
\left|e_{g}\right|=C_{v e}=\left(k_{g}^{*}+K_{\text {avg }}\left(\left|v_{o}^{*}\right|-\left|v_{o}\right|\right)\right) \omega_{g} / \omega_{\text {base }} .
$$

$k_{g}^{*}$ is the scalar quantity and the standard back EMF gain, $K_{a v g}$ is the output voltage regulator gain, $\left|v_{o}^{*}\right|[\mathrm{pu}]$ is the output voltage reference as a scalar quantity, and $\left|v_{o}\right|[\mathrm{pu}]$ is the magnitude of the output voltage. $\left|e_{g}^{*}\right|$ is a regulator that changes with time according to $\left|v_{o}\right|$ and keeps the output voltage at the target value.

$$
P_{i}=P_{o}^{*}+K_{g o v}\left(\omega_{\text {base }}-\omega_{g}\right)
$$


where, $K_{g o v}$ is the speed adjustment gain.

The input active power $P_{i}$ of the virtual generator is composed of the output power reference $P_{o}^{*}$ and the rotational speed governor component.

Assuming that $\omega_{g}, V_{o}, P_{o}$, and $P_{i}$ are constant as $\omega_{g k}, V_{o k}, P_{o k}$ and $P_{i k}$ during the sampling time $T_{s}$, the discrete-time system of Equation (20) to (25) can be expressed by the following equation.

$$
\begin{gathered}
\omega_{g k+1}=A_{v w} \omega_{g k}+B_{v w}\left(\tau_{i k}-\tau_{o k}\right) . \\
i_{v o k+1}=A_{v i} i_{v o k}+B_{v i}\left(e_{g k}-v_{o k}\right) . \\
e_{k+1}=A_{v e} e_{k} . \\
e_{g k}=C_{v e} e_{k} .
\end{gathered}
$$

where $\tau_{i k}$ and $\tau_{o k}$ are continuous-time scalar variables of $\tau_{i}$ and $\tau_{o}$ at discrete time at $k$, and $V_{o k}, e_{g h} i_{v o k}$ and $e_{k}$ are continuous-time vectors of $V_{o}, e_{g}, i_{v o}$, and $e$ at time $k . A_{v w} A_{v i}$ and $A_{v e}$ are the transition matrices obtained by differential Equations (20), (21), and (22), respectively, and are defined by the following equations.

$$
A_{v w}=\exp \left\{-T_{S} T_{g}^{-1}\right\}, A_{v i}=\exp \left\{-T_{s} T_{v o}^{-1}\right\}, A_{v e}=\exp \left\{-T_{s} \omega_{g k} S_{\alpha \beta}\right\}
$$

According to the differential equation solution formula, $B_{V W}$ and $B_{v i}$ are defined as follows.

$$
\begin{aligned}
& B_{v w}=\left\{\int_{0}^{T_{s}} \exp \left\{-\left(T_{s}-\xi\right) T_{g}^{-1}\right\} \mathrm{d} \xi\right\} \omega_{\text {base }} M_{g}^{-1} \\
& B_{v i}=\left\{\int_{0}^{T_{s}} \exp \left\{-\left(T_{s}-\xi\right) T_{v o}^{-1}\right\} \mathrm{d} \xi\right\} l_{g}^{-1}
\end{aligned}
$$

\subsection{Discreate-Time Model Following Controller under Constrains}

Figure 3 shows a system diagram of discrete-time model tracking control.

The control system is a hybrid system composed of three elements. This system is composed of three blocks: a continuous-time full-bridge inverter circuit connected to the grid system via an LCL filter, a discrete-time virtual generator model, and a state feedback system.

The virtual generator model is a time-varying vibration system. The model is not reachable. Therefore, the control system is derived by dividing the state space of the control system into reachable space and the non-reachable space.

The overall system equation is given by

$$
\begin{gathered}
x_{k+1}=A x_{k}+B v_{i k} . \\
{\left[\begin{array}{c}
i_{i k} \\
z_{k-1}
\end{array}\right]=C x_{k} .}
\end{gathered}
$$

Equation (32), the state equation, is divided into reachable space and others as follows.

$$
\left[\begin{array}{c}
x_{1 k+1} \\
X_{2 k+1}
\end{array}\right]=\left[\begin{array}{cc}
A_{11} & A_{12} \\
0 & A_{22}
\end{array}\right]\left[\begin{array}{c}
x_{1 k} \\
X_{2 k}
\end{array}\right]+\left[\begin{array}{c}
B_{1} \\
0
\end{array}\right] v_{i k} .
$$




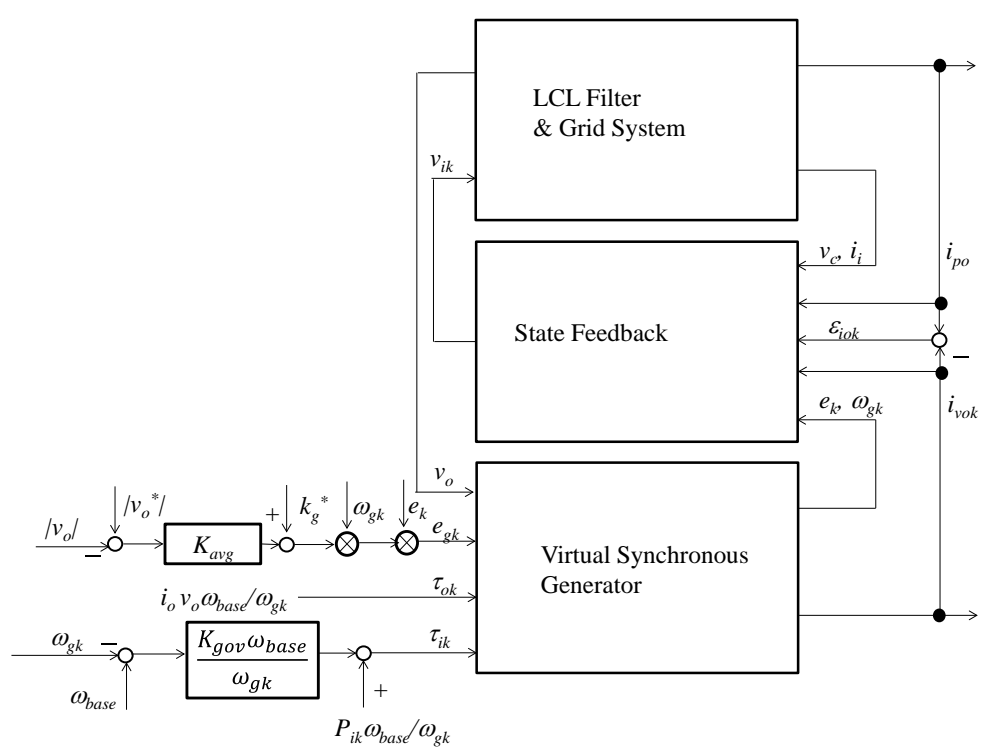

Figure 3. Discreate-time model following control using provided virtual synchronous generator.

Here, the state vector of the reachable space is $x_{1 k}=\left[x_{p 1 k}^{\mathrm{T}} z_{k-1}^{\mathrm{T}} i_{\text {vok }}^{\mathrm{T}}\right]^{\mathrm{T}}$, and the other state vectors are $x_{2 k}=\left[x_{c 2 k c}^{\mathrm{T}} v_{k}^{\mathrm{T}}\right]^{\mathrm{T}} ; \quad x_{p 1 k}=\left[i_{p o k}^{\mathrm{T}} v_{c k}^{\mathrm{T}} i_{i k}^{\mathrm{T}}\right]^{\mathrm{T}}$ and

$$
\begin{gathered}
x_{c 2 k}=\left[\omega_{g k} e_{k}^{\mathrm{T}}\right]^{\mathrm{T}} . \\
A_{11}=\left[\begin{array}{cc}
A_{p d 11} & 0 \\
A_{c 101} & A_{c 11}
\end{array}\right], A_{12}=\left[\begin{array}{cc}
0 & A_{p d 12} \\
A_{c 12} & A_{c 102}
\end{array}\right], B_{1}=\left[\begin{array}{c}
B_{p d 1} \\
0
\end{array}\right], A_{22}=\left[\begin{array}{cc}
A_{c 22} & 0 \\
0 & A_{p v g}
\end{array}\right] .
\end{gathered}
$$

where, $i_{p o k}=C_{p d 1} x_{p 1 k}, \quad z_{k}=A_{z} z_{k-1}+\left(i_{p o k}-i_{v o k}\right)$

$$
\begin{aligned}
& A_{z}=\exp \left\{T_{s} \omega_{g k} S_{\alpha \beta}\right\}, A_{c 101}=\left[\begin{array}{c}
C_{p d 1} \\
-B_{v i} C_{o 1}
\end{array}\right], A_{c 102}=\left[\begin{array}{c}
0 \\
-B_{v i} C_{o 2}
\end{array}\right] \\
& A_{c 11}=\left[\begin{array}{cc}
-A_{z} & -1 \\
0 & A_{v i}
\end{array}\right], A_{c 12}=\left[\begin{array}{cc}
0 & 0 \\
0 & B_{v i} C_{v e}
\end{array}\right], A_{c 22}\left(\omega_{g k}\right)=\left[\begin{array}{cc}
A_{v w} & 0 \\
0 & A_{v e}
\end{array}\right]
\end{aligned}
$$

( 0 in the above equation is a zero matrix, 1 is a unit matrix)

We have the quadratic evaluation function as follows

$$
J=\sum_{k=0}^{\infty}\left(x_{k}^{\mathrm{T}} Q x_{k}+x_{k}^{\mathrm{T}} S u_{k}+u_{k}^{\mathrm{T}} R u_{k}\right)
$$

where, state weight $Q$, state operation weight $S$ and operation weight $R$ are given by positive definite matrix.

In this case, the optimal input is:

$$
v_{i k}=-\left(R+B^{\mathrm{T}} P B\right)^{-1}\left(B^{\mathrm{T}} P A+S^{\mathrm{T}}\right) x_{k}
$$

where $P$ is the solution of the following discrete-time Riccati equation:

$$
P=A^{\mathrm{T}} P A+C^{\mathrm{T}} Q C-\left(A^{\mathrm{T}} P B+S\right)\left(R+B^{\mathrm{T}} P B\right)^{-1}\left(B^{\mathrm{T}} P A+S^{\mathrm{T}}\right)
$$

where, the matrix $P$ is divided into small matrices. 


$$
P=\left[\begin{array}{ll}
P_{11} & P_{12} \\
P_{12}^{T} & P_{22}
\end{array}\right]
$$

where $Q=\operatorname{diag}\left[Q_{11}, Q_{22}\right], C=\left[C_{1}, C_{2}\right]$.

Since the lower submatrix of the $B$ matrix in Equation (32) is a zero matrix, Equation (38) becomes as follows.

$$
v_{i k}=-\left(R+B_{1}^{\mathrm{T}} P_{11} B_{1}\right)^{-1}\left(\left(B_{1}^{\mathrm{T}} P_{11} A_{11}+S_{1}^{\mathrm{T}}\right) x_{1 k}+B_{1}^{\mathrm{T}}\left(P_{11} A_{12}+P_{12} A_{22}+S_{2}^{\mathrm{T}}\right) x_{2 k}\right)
$$

Therefore, if the solutions of $P_{11}$ and $P_{12}$ satisfying the following equations are obtained, it is enough to obtain Equation (43).

Since $A_{11}$ and $B_{1}$ are reachable, there is $P_{11}$ that satisfies Equation (42).

$$
P_{11}=A_{11}^{\mathrm{T}} P_{11} A_{11}+C_{1}^{\mathrm{T}} Q_{11} C_{1}-\left(A_{11}^{\mathrm{T}} P_{11} B_{1}+S_{1}\right)\left(R+B_{1}^{\mathrm{T}} P_{11} B_{1}\right)^{-1}\left(B_{1}^{\mathrm{T}} P_{11} A_{11}+S_{1}^{\mathrm{T}}\right)
$$

$P_{12}$ can also be obtained from the following equation.

$$
\begin{aligned}
P_{12}= & A_{11}^{\mathrm{T}}\left(P_{11} A_{12}+P_{12} A_{22}\right) \\
& -\left(A_{11}^{\mathrm{T}} P_{11} B_{1}+S_{1}\right)\left(R+B_{1}^{\mathrm{T}} P_{11} B_{1}\right)^{-1}\left(B_{1}^{\mathrm{T}}\left(P_{11} A_{12}+P_{12} A_{22}\right)+S_{2}^{\mathrm{T}}\right) .
\end{aligned}
$$

Since the virtual generator model is a time-varying system, the optimal state feedback gain is derived for each sampling time.

\section{Simulation Result}

The rated output of the virtual generator model is $1.2 \mathrm{MVA}$, the rated voltage is $400 \mathrm{~V}$, and the rated frequency of the system is $50 \mathrm{~Hz}$. The synchronous impedance is $2.0 \mathrm{pu}$, the synchronous impedance resistance component is $7.5 \times 10^{-2} \mathrm{pu}$ $(0.01 \Omega)$, the inertia constant is $3.5 \mathrm{sec}$, and the mechanical time constant is 100 sec because the friction loss is assumed to be small. The initial internal phase difference angle $\cos \delta=0.83$ and the reference back electromotive force $k_{g}^{*}: 1.2$. Speed adjustment gain $K_{\text {gor }}: 1 / 0.05 \omega_{\text {base }}$, voltage adjustment gain $K_{\text {avg: }}: 0.5$.

\subsection{Optimized Weight of Evaluation Function}

The weight of the evaluation function of the optimal control system is optimized by a multi-objective genetic algorithm, where, the operation weight $R=r 1$, the state weight is $C^{T} Q C=\operatorname{diag}\left[\begin{array}{lllll}0 & q_{i i} & q_{e r r} & 1 & 0\end{array}\right]$, the filter input current weight $q_{i i}$, and the error weight $q_{\text {err }}$ (" 1 " is unit matrix, " 0 " is zero matrix).

$$
S=\left[\begin{array}{l}
S_{1} \\
S_{2}
\end{array}\right]
$$

The weight $s_{q}$ is for the reactive power of the input and the weight for the active power is $s_{p}$, and the lower submatrix $S_{2}$ is defined as a zero matrix.

$$
S_{1}=s_{p} S_{\alpha \beta}+s_{p} 1
$$

( 1 in the above equation is the unit matrix)

We define the following objective function to be minimized for searching for Pareto optimal parameters using a multi-objective genetic algorithm.

$<<$ Object function $>>$ 
1) Average root-mean-square of the error current

2) Maximum peak of the filter input voltage

3) Maximum peak of the filter input current

4) Inductance of the inverter side

5) Inductance of the grid side

6) Capacitance of the filter

$$
\begin{aligned}
& \sqrt{\sum_{k=1}^{\infty} \varepsilon_{i o k}^{\mathrm{T}} \varepsilon_{i o k}}[\mathrm{pu}] \\
& \max _{k}\left|v_{i k}\right| \quad[\mathrm{pu}] \\
& \max _{k}\left|i_{i k}\right|[\mathrm{pu}] \\
& L_{L C L 1}[\mathrm{H}] \\
& L_{L C L 2}[\mathrm{H}] \\
& C_{L C L}[\mathrm{~F}]
\end{aligned}
$$

where, the output current error is given by the following equation.

$$
\varepsilon_{\text {iok }}=i_{p o k}-i_{v o k}
$$

The primary purpose of the control is to minimize the error between the output current and the model output current. The purpose of this evaluation is (1).

On the other hand, a trade-off relationship is expected between the error current and the filter input voltage. Therefore, at the same time, (2) is added as the purpose for minimizing the input voltage.

The purpose (3) is to minimize the output of the inverter because the capacity of the inverter is limited.

In the proposed inverter, a filter is placed between the grid system and the inverter optimal controlled for an arbitrary system. Cost reduction can be expected if the inductance and capacitance of the designed filter are small. For this purpose, objectives (4) to (6) are added.

In consideration of the above, we solve the optimization problem with $8 \mathrm{ob}$ jective functions.

The following eight design variables are applied to multi-objective genetic optimization as genes.

$<<$ Gene $>>$
1) Inductance of the inverter side
$L_{L C L 1}[\mathrm{H}]$
2) Inductance of the grid side
$L_{L C L 2}[\mathrm{H}]$
3) Cut-off frequency
$\omega_{\text {cutoff }}[\mathrm{rad} / \mathrm{sec}]$
4) Error weight
$q_{\text {err }}$
5) Weight of the filter input current
$q_{i i}$
6) Weight of the filter input voltage
$r$
7) Weight of the filter input reactive power $s_{q}$
8) Weight of the filter input active power
$s_{q}$

Individual with large errors are not design solutions that can be selected, so individual with errors exceeding $10 \%$ of the rating are rejected. Individual with a maximum input current exceeding 60 [pu] are discarded. Since the cut-off frequency is based on the rated frequency and the switching frequency is $7.4 \mathrm{kHz}$, solutions of 74th order or higher are discarded.

Based on [11], we have used the following genetic algorithm to solve the above multi-objective optimization problem.

Step 1: Initialize

Give the individual $\dot{\boldsymbol{i}}_{i}$ (60 individuals) as element of the external set $\bar{I}$ and the population set $I$.

Step 2: Fitness assignment 
The fitness $F\left(\boldsymbol{i}_{i}\right)$ of individual $\boldsymbol{i}_{i}$ is following equation:

$$
F\left(\boldsymbol{i}_{i}\right)=\sum_{i 1 \neq i, i_{i 1} \succ \boldsymbol{i}_{i}}^{\text {all }} S\left(\boldsymbol{i}_{i 1}\right)
$$

where the strength $S\left(i_{i}\right)$ of individual $I_{i}$ is the number of solutions it dominates:

$$
S\left(\boldsymbol{i}_{i 1}\right)=\operatorname{num}\left(\left\{\boldsymbol{i}_{i 2} \mid \boldsymbol{i}_{i 1} \succcurlyeq \boldsymbol{i}_{i 2}\right\}\right)
$$

where the number of elements of the set $A$ is expressed as num $(A)$ and the equation $\boldsymbol{i}_{i 1} \succcurlyeq \boldsymbol{i}_{i 2}$ represent the relation of weak pareto dominance:

$$
\left(O_{j 1}\left(\boldsymbol{i}_{i 1}\right) \leq O_{j 1}\left(\boldsymbol{i}_{i 2}\right)\right) \wedge\left(O_{j 2}\left(\boldsymbol{i}_{i 1}\right)<O_{j 2}\left(\boldsymbol{i}_{i 2}\right)\right)(\forall j 1, \exists j 2,(j 1, j 2 \in\{1, \cdots, 6\}))
$$

Step 3: Selection

Delete one of the closest individuals in the objective function space. Good individuals are selected for the next generation of population set using binary tournament selection.

Step 4: Recombination and mutation operators

Step 5: Algorithm termination judgment

Return to step 2, if not the last generation (60 generations).

$O_{j}\left(I_{i}\right)$ is the objective function with objective number $j$, the $i$ th individual with a set of genes $\{1 . .8\}$ is $\boldsymbol{i}_{i}$.

Here, objective functions (1) to (3) were evaluated by Runge-Kutta method for the initial time-varying of each individual (design solution) in the ideal hybrid system of continuous time system (solution time step: $0.01 \mathrm{Ts)} \mathrm{and} \mathrm{discrete} \mathrm{time}$ system with sample time $T_{s}$.

Figure 4 shows the Pareto optimized weight distribution.

The markers in Figure 4 represent individual design solutions; square markers represent good design solutions, and triangular markers represent top $10 \mathrm{de}-$ sign solutions.

Referring to Figure 4(a), individuals are gathered in a straight line near the same weight and individuals with lower maximum filter input voltage are not born. Collected weight is considered to be the optimal value of the weight $r$. Looking at the distribution of the error weight and the filter input current weight, it can be seen that the optimal design solution can be selected at one place (Figure 4(b)).

The filter input current tends to increase as the error weight increases and the output current error decreases. It seems that individuals are gathering to one place where the input current increases by increasing the error weight and the effect of decreasing the input current by increasing the input current weight is cancelled each other.

On the other hand, the active power weight and the reactive power weight are concentrated in one place, but the distribution is wide (Figure 4(c)). From this result, it is considered that the objective function affecting $s_{q}$ snd $s_{p}$ is insufficient.

Figure 4(d) shows the Pareto optimal solution distribution of the cut-off frequency and the maximum filter input current. 


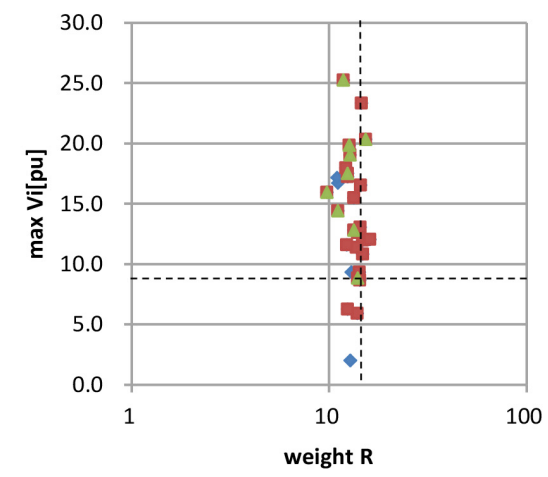

(a) weight $\mathrm{R}$ for maximum peak of filter input voltage

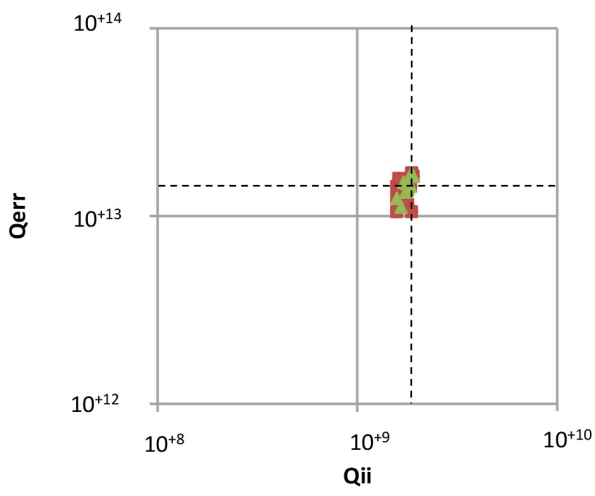

(b) weight qerr vs weight qii

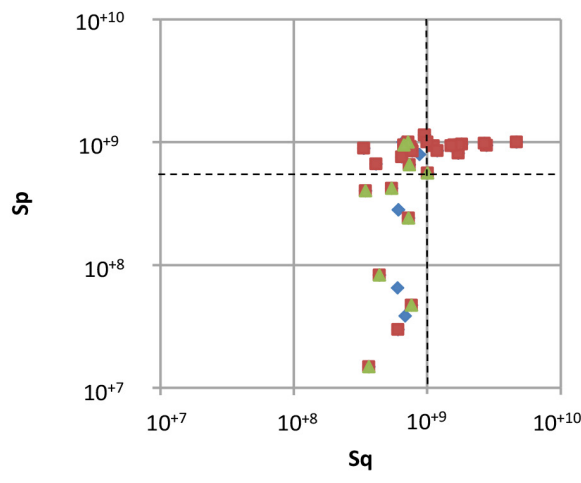

(c) weightSp vs weight Sq.

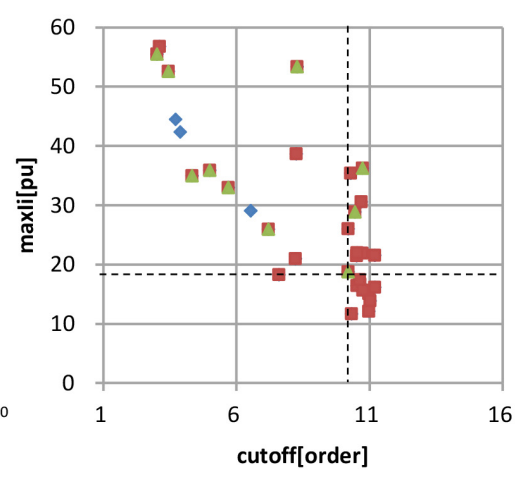

(d) Pareto optimal LCL filter

Figure 4. Pareto-optimal set of weight parameter.

From the figure, it can be seen that the higher the cut-off frequency, the lower the peak current becomes in the Pareto curve. The absence of a design solution of order 11 or higher is considered to be the result of inferior solution discard of large error current.

\subsection{Output Transient Response}

Figure 5 shows the results of the transient response of the output power in the numerical analysis using the Pareto-optimized load described in the previous section.

From the Pareto optimal solution, the filter and load are $\omega_{\text {base }} L_{L C L 1}: 2.36 \times 10^{-2}$ $\mathrm{pu}$, $\omega_{\text {base }} L_{L C L 2}: 4.41 \times 10^{-2} \mathrm{pu}$, cut-off frequency $10.2 \times \omega_{\text {base }}[\mathrm{rad} / \mathrm{sec}]$, error weight $q_{\text {err }}: 1.44 \times 10^{13}$, input current weight $q_{i i}: 1.86 \times 10^{9}$, input voltage weight $r$. 14.0, active power weight $s_{p}: 5.60 \times 10^{8}$, reactive power weight $s_{q}: 1.01 \times 10^{9}$. Figure 5 shows the design solutions selected at the intersections of the dotted lines of Figure 4. In the simulation time sequence, the input of the virtual synchronous generator stepped down from $1 \mathrm{pu}$ to $0 \mathrm{pu}$ in $4 \mathrm{sec}$, and stepped up from 0 pu to $1 \mathrm{pu}$ after 1 second, assuming the worst case of power fluctuation of the wind generator.

Since the input of the model changes, the output of the inverter also fluctuates step by step (Figure 5(b), Figure 5(c)). However, the error waveform shows that 


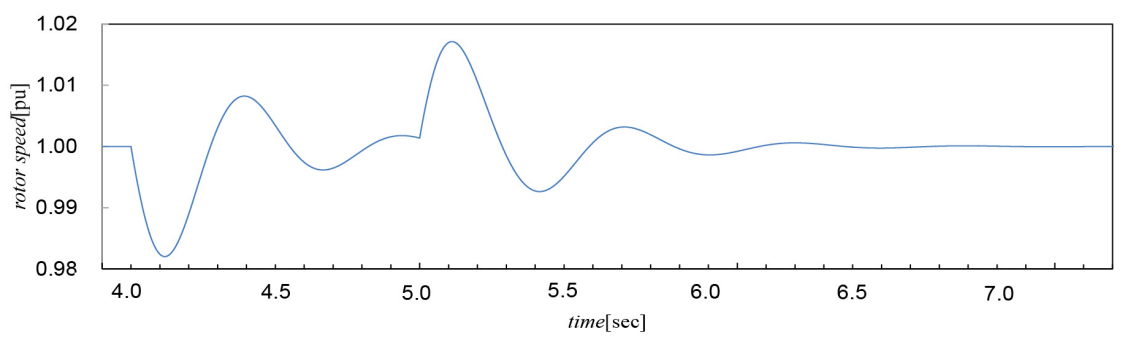

(a) rotor speed

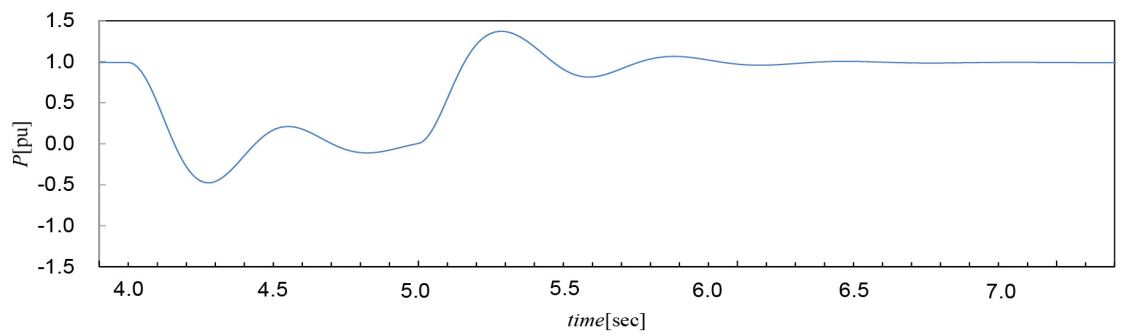

(b) output power

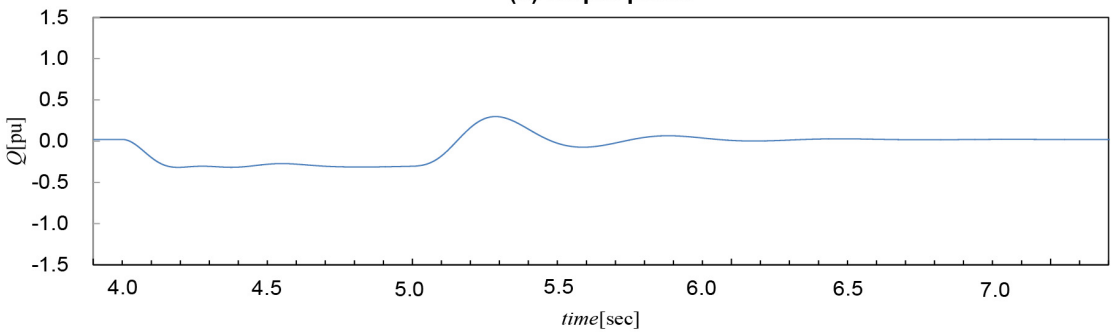

(c) reactive power

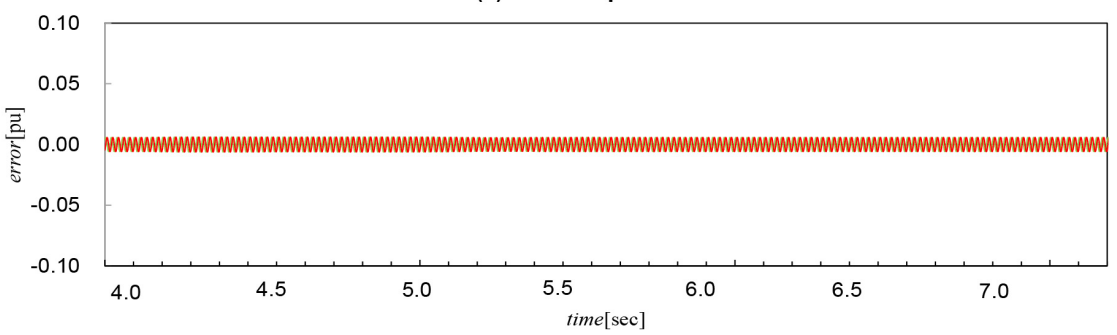

(d) output current error (blue line: zero-phase, green line: positive-phase, red line: negative phase)

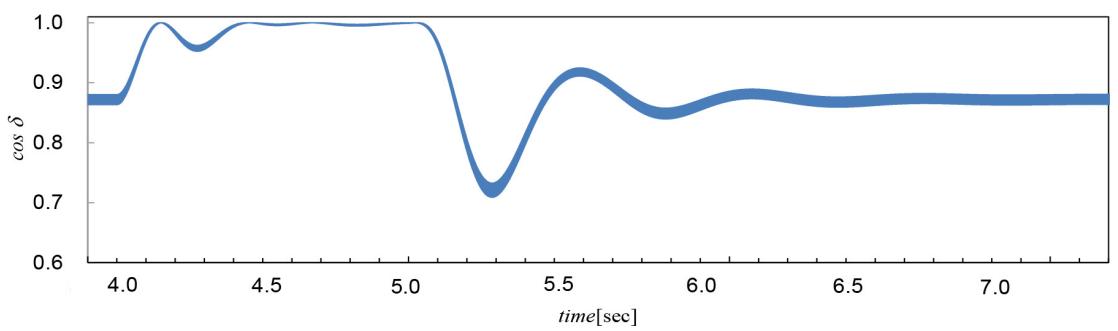

(e) internal phase cos delta

Figure 5. Transient response of the proposed method.

the waveform follows the target waveform with an error of $0.5 \%$ or less (Figure 5(d)). The inertia of the virtual synchronous generator also suppresses the low speed of the rotor speed (Figure 5(a)). The internal phase angle delta in the proposed method is within the range of 0 to $30 \mathrm{deg}$ (Figure 5(e)). Therefore, it can be said that the proposed model behaves well as a virtual synchronous generator. 


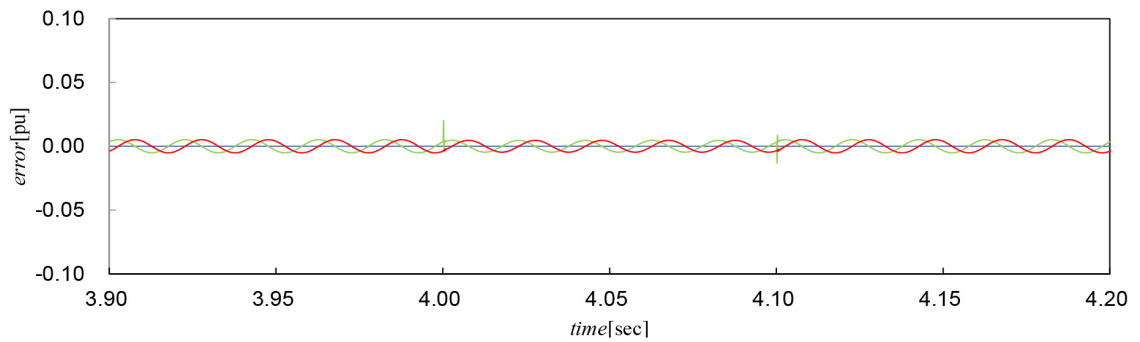

(a) Output current error (blue line: zero-phase, green line: positive-phase, red line: negative phase)

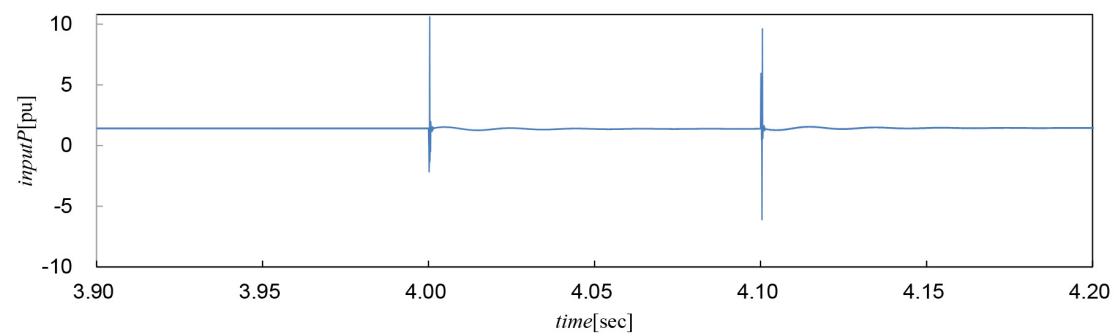

(b) Active power of the inverter side filter

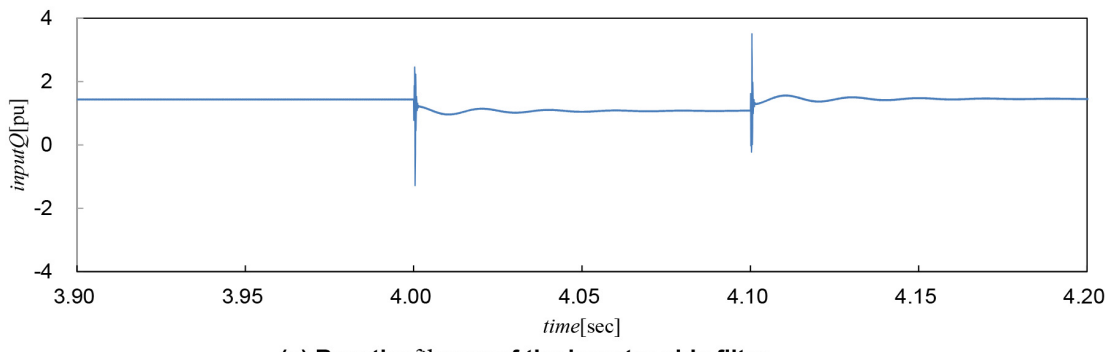

(c) Reactive power of the inverter side filter

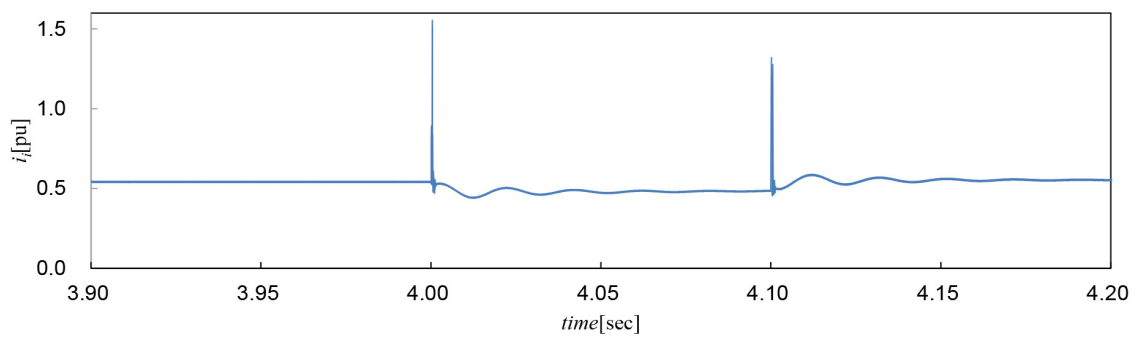

(d) Inverter side current of the filter

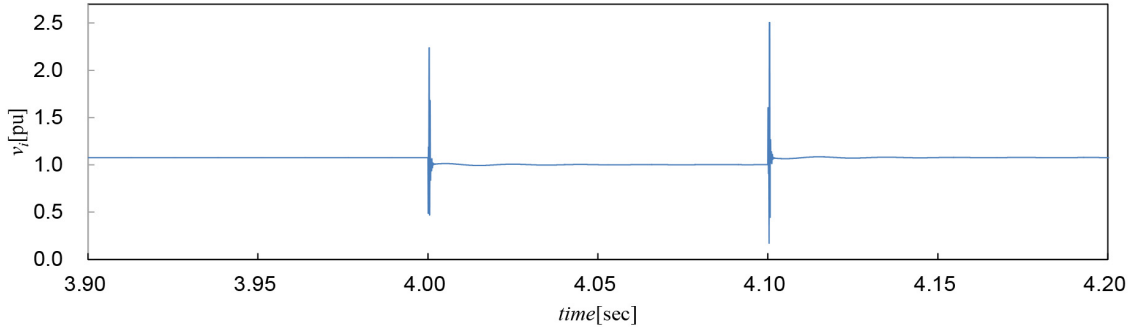

(e) Inverter side voltage of the filter

Figure 6. Instantaneous voltage drop response without weight $q_{i i}, s_{p}$ and $s_{q}$.

\subsection{Instantaneous Grid Voltage Drop}

The results of the instantaneous voltage drop using the Pareto-optimized load described in the previous section are shown.

In the sequence of the instantaneous voltage drop, it is assumed that the voltage drops by $10 \%$ at 4 seconds and returns after 0.1 seconds. The system 


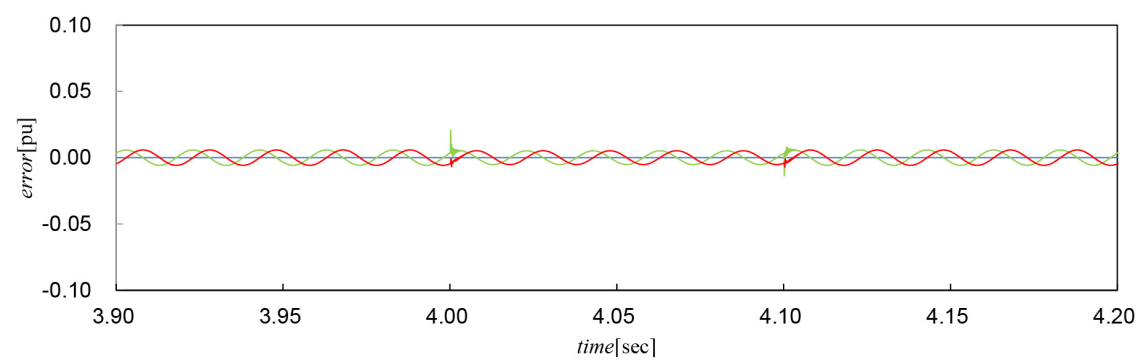

(a) Output current error (blue line: zero-phase, green line: positive-phase, red line: negative phase)

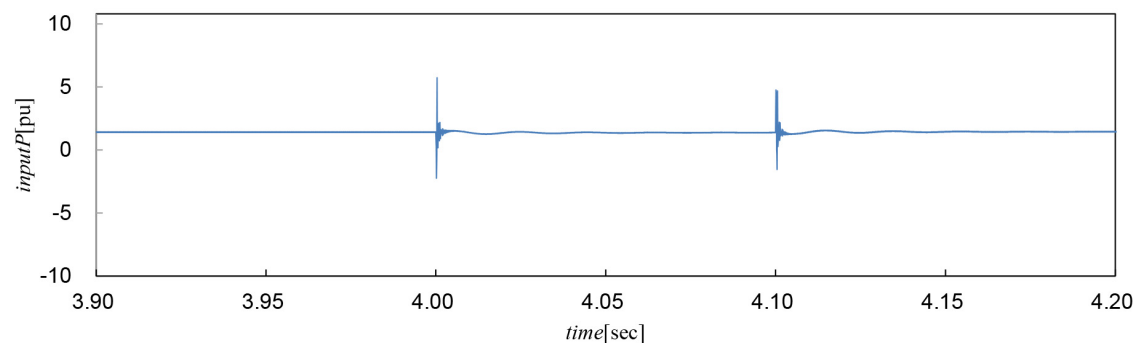

(b) Active power of the inverter side filter

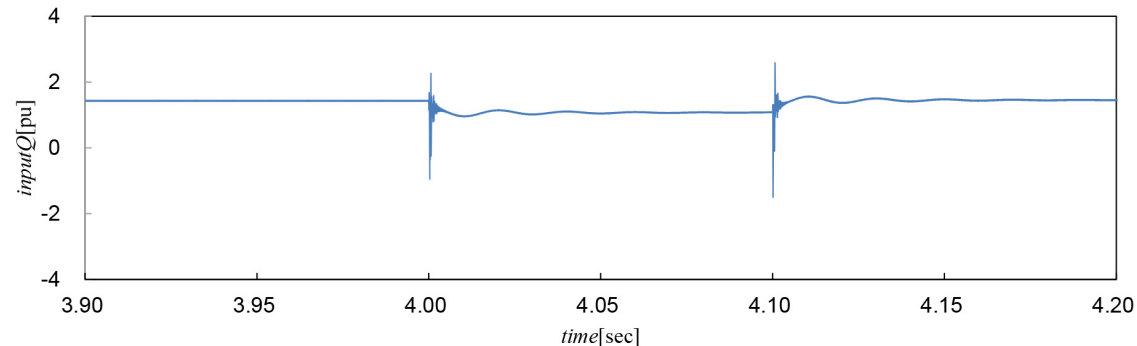

(c) Reactive power of the inverter side filter

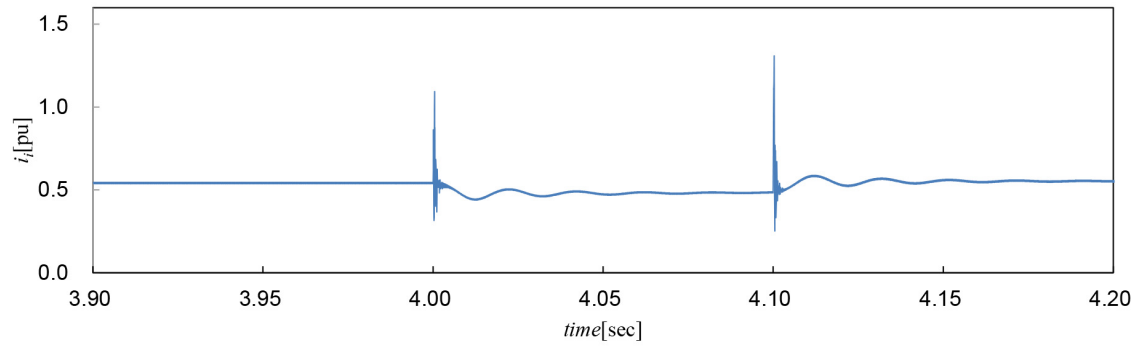

(d) Inverter side current of the filter

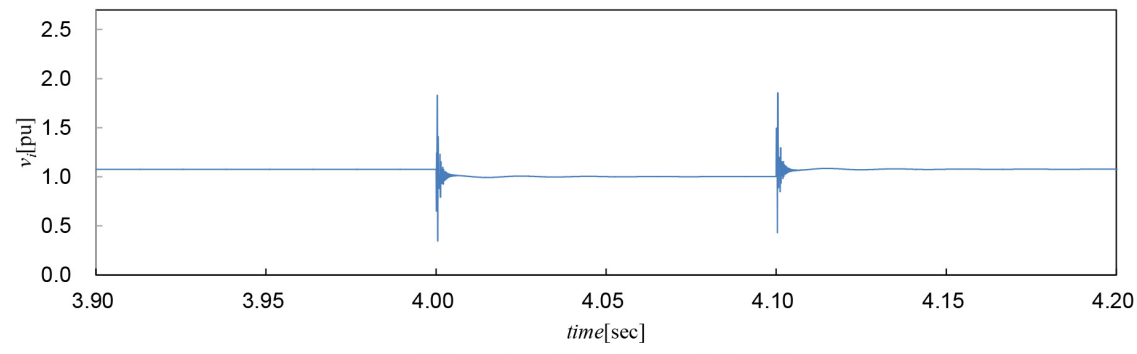

(e) Inverter side voltage of the filter

Figure 7. Instantaneous voltage drop response with weight $q_{i i}, s_{p}$ and $s_{q}$.

parameters are the same as in the previous section. Figure 6 shows the results when there is no filter input current weight, active power weight, and reactive power weight, and Figure 7 shows the results when there are the weights.

Figure 6(a) and Figure 7(a) show error current. The error is less than 1\%, 
and it can be said that both responses follow the model well.

As seen from Figure 6(b) and Figure 7(b), the output fluctuates due to the voltage drop, but the peak is suppressed to half. It can be seen that the peaks of the reactive power, input current and input voltage to the filter are somewhat suppressed by the weight of the evaluation function.

The maximum peak was 3.5pu without weight (Figure 6(c)), but was reduced to $2.58 \mathrm{pu}$ (Figure $7(\mathrm{c})$ ). The maximum peak of the input current was $1.55 \mathrm{pu}$ without weight (Figure 6(d)), but was suppressed to $1.30 \mathrm{pu}$ due to the weight (Figure $7(\mathrm{~d})$ ). Although the input voltage is not directly related to the weight $q_{i i}$ $s_{p}$, and $s_{q}$, it was affected by the weight, and the maximum was $2.50 \mathrm{pu}$, but was reduced to $1.85 \mathrm{pu}$ (Figure 6(e), Figure $7(\mathrm{e})$ ).

It is shown that the inverter rating can be reduced by optimizing the load of the evaluation function while achieving the same performance.

\section{Conclusions}

In this paper, as a current control method for a virtual generator model with synchronization power, we proposed the application of discrete-time model following control considering output suppression by optimizing the gain of a multivariable evaluation function.

Since the proposed control method uses instantaneous value control, it can follow the virtual generator model well.

Since a multivariable evaluation function was used, not only the tracking error but also the suppression of the input voltage and input current from the inverter device to the filter could be considered in the control system.

In this control method, good tracking performance and output suppression can be expected by deriving the feedback gain for each sample time even when the frequency fluctuation is large.

Therefore, it is concluded that the proposed method is useful for realizing virtual generator models that contribute to enhancing the grid system stability.

Grid stabilization depends on the virtual synchronous generator model performance. As a future study, we are planning to propose new design method for the condition of output suppression in the virtual generator model supporting and stabilizing the grid system.

\section{Acknowledgements}

Supported by JSPS KAKENHI Grant Number JP17K06289.

\section{Conflicts of Interest}

The authors declare no conflicts of interest regarding the publication of this paper.

\section{References}

[1] Global Wind Energy Council (GWEC) (2019) Global Wind Report 2018. 
[2] Agency for Natural Resources and Energy, Feed-In Tariff System Website for Information Disclosure, 2020. http://www.fit-portal.go.jp/PublicInfoSummary

[3] Yin, X.X. (2018) Hybrid Adaptive Control for Variable-Speed Variable-Pitch Wind Energy Systems Using General Regreeion Neural Network. Wind Energy Science Discussions, 18 p. https://doi.org/10.5194/wes-2018-19

[4] Tiwari, R. and Babu, N.R. (2019) Artificial Network-Based Control Strategies for PMCG-Based Connected Wind Energy Conversion System. International Journal of Materials and Product Technology, 58, 323-341. https://doi.org/10.1504/IJMPT.2019.100009

[5] Bevrani, H. (2014) Robust Power System Frequncy Control. Second Edition, Springer, Berlin, 388 p. https://doi.org/10.1007/978-3-319-07278-4

[6] Torres, M. and Lopes, L.A.C. (2013) Virtual Synchronous Generator: A Control Strategy to Improve Dynamic Frequency Control in Autonomous Power Systems. Energy and Power Engineering, 5, 32-33. https://doi.org/10.4236/epe.2013.52A005

[7] Poolla, B.K., Grob, D. and Dorfler, F. (2019) Placement and Implementation of Grid-Forming and Grid-Following Virtual Inertia and Fast Frequency Response. IEEE Transaction on Power Systems, 34, 3035-3046. https://doi.org/10.1109/TPWRS.2019.2892290

[8] Umemura, A., Takahashi, R. and Tamura, J. (2019) The Discrete-Time Model Following Controller for a Virtual Synchronous Generator. IEEE Asia-Pacific Power and Energy Engineering Conference (APPEEC 2019), Macao, 1-4 December 2019, 1-6. https://doi.org/10.1109/APPEEC45492.2019.8994476

[9] Guo, Q., Wang, Q. and Liu, Y.L. (2018) Antiwindup Control of an Electrohydraulic System with Load Disturbance and Modeling Uncertainty. IEEE Transactions on Industrial Informations, 14, 3097-3108. https://doi.org/10.1109/TII.2017.2768106

[10] us Saqib, N., Rehan, M., Iqdal, N. and Hong, K.-S. (2018) Static Antiwindup Design for Nonlinear Parameter Varying Systems with Application to DC Motor Speed Control under Nonlinearities and Load Variations. IEEE Transactions on Control Systems Technology, 26, 1091-1098. https://doi.org/10.1109/TCST.2017.2692745

[11] Bleular, S., Brack, M., Thiele, L. and Zitzler, E. (2001) Multiobjective Genetic Programming: Reducing Bloat Using SPEA2. Proceedings of the 2001 Congress on Evolutionary Computation, Seoul, 27-30 May 2001, 536-543.

https://doi.org/10.1109/CEC.2001.934438 


\section{Nomenclature}

$V_{\text {inva }}, V_{\text {invb }}, V_{\text {invc }}$ the filter input voltage (a-b-c phase) [V]

$L_{L C L L}$ : the inverter-side inductance of the LCL filter [H]

$R_{L C L 1}$ : the inverter-side inductance loss of the LCL filter $[\Omega]$

$C_{L C L}$ : the star connected capacitance of the LCL filter [F]

$L_{L C L 2}$ : the grid-side inductance of the LCL filter $[\mathrm{H}]$

$R_{L C L 2}$ : the grid-side inductance loss of the LCL filter $[\Omega]$

$L_{\text {grid. }}$ the grid system inductance component $[\mathrm{H}]$

$R_{\text {grid. }}$ the grid system loss $[\Omega]$

$V_{\text {grida, }} V_{\text {gridb }}, V_{\text {gridc: }}$ the grid system voltage (a-b-c phase) [V]

$V_{c Y a}, V_{c Y b}, V_{c Y c}$ the capacitance voltage (a-b-c phase) [V]

$V_{\text {outa, }}, V_{\text {outb }}, V_{\text {outc: }}$ the output voltage of the LCL filter (a-b-c phase) [V]

$I_{\text {inva }} I_{\text {invb }}, I_{\text {invc: }}$ the input current of the LCL filter (a-b-c phase) [A]

$I_{\text {outa }} I_{\text {outb }}, I_{\text {outc }}$ the output current of the LCL filter (a-b-c phase) [A]

$S_{\text {base }}$ the rated capacity of the virtual generator [VA]

$V_{\text {baseY }}$ the rated voltage of the virtual generator [V]

$\omega_{\text {base }}$ the rated system frequency $[\mathrm{rad} / \mathrm{sec}]$

$\omega_{\text {cutoff: }}$ the cutoff frequency of the LCL filter $[\mathrm{rad} / \mathrm{sec}]$

$T_{s}$ : sampling time [sec]

$k$. discrete-time

$C_{\alpha \beta}^{a b c}$ : the three-phase to two-phase conversion matrix

$i_{p o}$ : the normalized LCL filter output current vector $[\mathrm{pu}]$

$i_{i}$ : the normalized LCL filter input current vector [pu]

$v_{c}:$ the normalized LCL filter capacitance voltage vector [pu]

$v_{o}$ : the normalized LCL filter output voltage vector [pu]

$\mathrm{V}_{\mathrm{g}}$ : the normalized grid system voltage vector [pu]

$v$ : the grid system voltage unit vector

$v_{i}:$ the normalized LCL filter input voltage vector [pu]

$T_{g d}$ : the time constant matrix of the grid system [sec]

$l_{g d i}$ : the inductance matrix of the grid system

$T_{1}$ : the time constant matrix of the inverter-side LCL filter [sec]

$I_{1}$ : the inductance matrix of the inverter-side LCL filter

$T_{2}$ : the time constant matrix of the grid-side LCL filter [sec]

$l_{2}$ : the inductance matrix of the grid-side LCL filter

$c_{Y}$ : the capacitance matrix of the LCL filter

$\omega_{g}$ : the rotation speed of the virtual generator $[\mathrm{rad} / \mathrm{sec}]$

$P_{i}, P_{o}$ : the input power and the output power of the virtual generator [pu]

$P_{o}^{*}$ : the output power reference of the virtual generator $[\mathrm{pu}]$

$\tau_{i}, \tau_{o}$ : the input torque and the output torque of the virtual generator [pu]

$T_{g}$ : the time constant of the virtual generator [sec]

$M_{g}$ : the inertia constant of the virtual generator [sec]

$i_{v o}$ : the output current vector of the virtual generator $[\mathrm{pu}]$

$e_{g}$ : the normalized no-load voltage vector of the virtual generator [pu] 
$e$ : the no-load voltage unit vector of the virtual generator [pu]

$T_{v o}$ : the time constant matrix of the virtual generator [sec]

$l_{g}$ : the inductance matrix of the virtual generator

$k_{g}^{*}$ : the standard back EMF gain of the virtual generator

$K_{\text {avg: }}$ the voltage regulator gain of the virtual generator

$K_{\text {gov }}$ : the speed adjustment gain of the virtual generator

$\varepsilon_{\text {kiok: }}$ : the output current error discrete-time vector

$Z_{k}$ : the output current error evaluation state discrete-time vector

$q_{i i}$ : the filter input current weight

$q_{\text {err }}$ the error weight

$r$. the filter input voltage weight

$s_{p}$ : the filter input active power weight

$s_{q}$ : the filter input reactive power weight

I: the population set

$\overline{\boldsymbol{I}}$ : the external set

$i_{i}$ : the $i$ th individual (design solution)

$F\left(i_{i}\right)$ : the fitness function of the $i$ th individual

$S\left(\boldsymbol{i}_{i}\right)$ : the strength function of the $i$ th individual

$O_{f}\left(i_{i}\right)$ : the $j$ th objective function of the $i$ th individual 